\title{
Investigation of the role of ETGs in electron heat transport in TCV plasmas
}

\author{
A. Mariani, ${ }^{1, *}$ P. Mantica, ${ }^{1}$ S. Brunner, ${ }^{2}$ M. Fontana,${ }^{2}$ A. Karpushov, ${ }^{2}$ C. Marini, ${ }^{3}$ \\ L. Porte ${ }^{2}$ O. Sauter, ${ }^{2}$ the TCV Team, ${ }^{\dagger}$ and the EUROfusion MST1 Team \\ ${ }^{1}$ Istituto di Scienza e Tecnologia dei Plasmi, CNR, via Cozzi 53, 20125 Milan, Italy \\ ${ }^{2}$ École Polytechnique Fédérale de Lausanne (EPFL), Swiss Plasma Center (SPC), 1015 Lausanne, Switzerland \\ ${ }^{3}$ General Atomics, P.O. Box 85608, San Diego, California 92186
}

(Dated: August 1, 2019)

\begin{abstract}
Electron-scale micro-turbulence driven by the electron temperature gradient (ETG) instability have recently been shown to impact the electron heat transport in tokamaks. Given the relevance of these mechanisms for ITER scenarios, a new study has been carried out on the TCV tokamak at the Swiss Plasma Center, which is equipped with both ECH and NBI heating, allowing to investigate the relevance of ETG transport. Dedicated L-mode TCV discharges have been performed and the experimental measurements have been compared with gyrokinetic simulations. The results indicate that ETGs should contribute to electron heat transport from midradius to the edge when NBI and ECH are simultaneously applied, while the cases with pure ECH are dominated by ion-scale turbulence at mid-radius and show signs of possible ETG contribution only at outer radii.
\end{abstract}

\section{INTRODUCTION}

The understanding of heat transport in fusion plasmas is a key point towards the design of future reactors. The observed levels of transport in tokamak plasmas are mainly the result of drift-wave turbulence driven by the free energy available in the plasma density and temperature gradients [1]. In typical plasma core conditions, ion temperature gradient modes (ITG, driven dominantly by ion temperature gradient $\nabla T_{i}$ ) and trapped electron modes (TEM, driven dominantly by electron temperature and density gradients $\nabla T_{e}$ and $\left.\nabla n_{e}\right)$ are the most important micro-instabilities at ion scales $\left(k_{\theta} \rho_{i} \lesssim 1\right.$, where $k_{\theta}$ is the poloidal wave number and $\rho_{i}$ is the ion Larmor radius), while electron temperature gradient modes (ETG, driven dominantly by electron temperature gradient) are the main instabilities at electron scales $\left(k_{\theta} \rho_{e} \lesssim 1, \rho_{e}\right.$ the electron Larmor radius). All these instabilities present a threshold in the logarithmic temperature gradients $\left(\left|\nabla T_{i}\right| / T_{i}\right.$ and $\left|\nabla T_{e}\right| / T_{e}$ for ITG and TEM/ETG, respectively), followed by a monotonic increase of the linear growth rate and heat fluxes (ion and electron heat fluxes $q_{i}$ and $q_{e}$, respectively). The slope of $q_{i, e}$ versus $\left|\nabla T_{i, e}\right| / T_{i, e}$ is related to the level of stiffness of the transport, which characterises how strongly $T_{i, e}$ profiles are tied to the threshold.

While electron-scale isotropic eddies would imply negligible transport, numerical simulations of ETGs show radially elongated structures, known as ETG streamers [2], which by increasing the radial size of the turbulence could allow significant electron heat transport [3]. Indeed, from the experimental side, a correlation between the increase of the electron heat flux $q_{e}$ and the increase of high-k density fluctuations has been reported in [4-8]. Modelling plasmas featuring ETGs by means of Gyrokinetic (GK) [9] codes can give an insight

\footnotetext{
* Electronic address: mariani@ifp.cnr.it

$\dagger$ See author list of S. Coda et al. 2019 Nucl. Fusion accepted (https://doi.org/10.1088/1741-4326/ab25cb)

* See author list of B. Labit et al. 2019 Nucl. Fusion accepted (https://doi.org/10.1088/1741-4326/ab2211)
}

on the impact that they have on the turbulent transport. Due to the very high numerical cost of GK multi-scale simulations (i.e. simulations resolving both ion and electron scales), only the ion scales are accounted for in most studies. This simplified approach has been applied for example to electron heat transport experiments in JET [10], C-MOD [11] and DIII-D [12], and in some cases neither the $q_{e}$ values nor their stiffness were matched by low-k non-linear (NL) GK simulations. Moreover, a limited number of recent studies carried out with multi-scale NL GK simulations have shown that high-k instabilities and multi-scale interactions can have a strong impact on electron and ion heat fluxes, especially when low-k modes are close to marginal stability $[11,13-16]$.

The aim of this paper is to investigate the impact of ETGs on electron heat transport in plasmas of the Tokamak à Configuration Variable (TCV) [17] at the Swiss Plasma Center (SPC-EPFL, Lausanne, Switzerland). ETGs are destabilised by decreasing the value of $\tau=Z_{\mathrm{eff}} T_{e} / T_{i}$ [18], where $Z_{\mathrm{eff}}$ is the effective charge and $T_{e} / T_{i}$ the electron/ion temperature ratio. TCV is equipped with both ECH and NBI heating, allowing respectively to vary $\left|\nabla T_{e}\right|$ and $T_{e} / T_{i}$. This work consists of two parts: experimental analysis and gyrokinetic modelling. From the experimental side, two dedicated TCV discharges have been performed during the EUROfusion WP MST1 2017 experimental campaign, in order to characterise the $\left|\nabla T_{e}\right| / T_{e}$ threshold and stiffness of electron heat flux at mid-radius varying $\tau$, to test the compatibility of the results with an ETGdominated high-stiffness picture. The two discharges present on- and off-axis ECH power deposition respectively, each including different time intervals with different NBI/ECH power fractions (NBI only, ECH only and mixed NBI/ECH). Concerning the numerical analysis, in view of a future investigation to be carried out by means of NL multi-scale GK simulations, linear flux-tube multi-scale $k_{\theta}$ scans have been performed with the GENE code $[19,20]$ in order to compute the ETG thresholds for the considered cases, while NL ion-scale flux-tube simulations have been run to compare the results with the experimental $q_{e}$ values, to assess where the ion scales are sufficient to explain the experimental fluxes and stiffness and where a possible ETG contribution may need to be in- 
voked. The paper is organised as follows: in section II the experimental settings, together with the simulations parameters, are introduced. Sections III and IV illustrate the experimental analysis and GK modelling respectively. In particular, in section III A the steady state $\left|\nabla T_{e}\right| / T_{e}$ scan of the experimental electron heat flux at mid-radius is presented, comparing results corresponding to ECH deposition on- and off-axis, whilst section III B shows the perturbative analysis of the heat wave produced by ECH modulation, complemented with the analysis of the propagation of the sawtooth (ST) magnetohydrodynamic instability where the modulation data were not fully reliable due to the presence of large ST. In section IV A the linear GK results are shown, presenting the turbulence growth rate spectra at mean experimental parameters and the ETG linear $\left|\nabla T_{e}\right| / T_{e}$ thresholds. Finally, section IV B contains the NL GK results and compares them with experiments. Conclusions are drawn in section $\mathrm{V}$.

\section{EXPERIMENTAL CONDITIONS AND SIMULATION PARAMETERS}

Two TCV L-mode discharges are considered, with vacuum magnetic field $\mathrm{B}_{0}=1.41 \mathrm{~T}$ on the magnetic axis with major radius $\mathrm{R}=88 \mathrm{~cm}$ and plasma current $\mathrm{I}_{p}=170 \mathrm{kA}$, confining an electron-deuteron plasma with carbon as main impurity. Six phases are present in every shot, varying the proportion of $\mathrm{ECH} / \mathrm{NBI}$ power which is deposited in the plasma, from NBI only injection to ECH only (see Fig.1 (a)). ECH has been injected on-axis and off-axis, respectively, in the two discharges \#59113 and \#59112, in order to estimate for each phase with ECH the threshold and stiffness of the electron heat transport. ECH was injected both steady and modulated in different time intervals, in order to gain additional information from heat wave propagation analysis. In this work we consider three phases, labelled P1, P3, P5 in Fig.1 (a). The first has NBI only, the second NBI and ECH with high duty cycle (high-dc) modulation (167 Hz, with $4.5 \mathrm{~ms}$ on/1.5 ms off, see Fig.1 (b)), while the third has ECH only with high-dc modulation. For addressing the P5 phase with ECH off-axis the identical discharge \#59111 was considered instead of \#59112 because of a gyrotron failure in \#59112. The corresponding profiles of ECH average power density and NBI average power density to electrons are provided in Fig.1 (c) and (d) respectively. The NBI power takes into account duct, shine-through, orbit and charge exchange losses.

The electron density $n_{e}$ and temperature $T_{e}$ are measured experimentally with the Thomson scattering diagnostic, while the temperature $T_{c}$ is given by the charge exchange recombination spectroscopy (CXRS). The CXRS diagnostic employs a Diagnostic Neutral Beam Injector (DNBI), whose power is negligibly absorbed by the plasma, due to high shinethrough and high orbit losses, as tested by ASTRA [21] interpretative simulations. The corresponding quantities for ions are obtained by enforcing neutrality $\left(n_{i}=n_{e}-6 n_{c}\right)$ and deuteron-carbon thermal equilibrium $\left(T_{i}=T_{c}\right)$. The ion/carbon density proportion is constrained to the effective charge $Z_{\mathrm{eff}}=\sum_{j} Z_{j}^{2} n_{j} / n_{e}$ (the sum is on the ion species), ob-
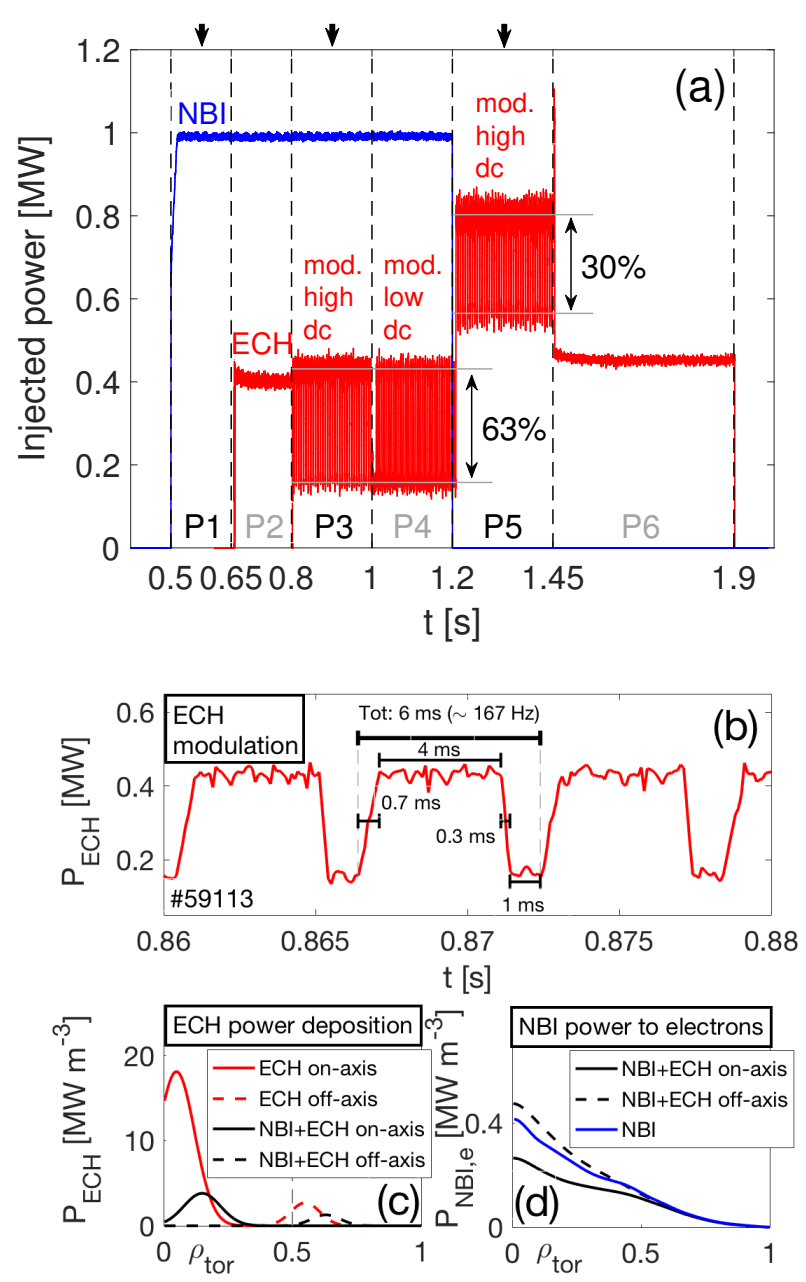

Figure 1. (color online) (a) NBI (blue) and ECH (red) total injected power time traces. P1-P6 indicate the six different phases of each discharge. The black arrows on top of the figure indicate the three phases that have been considered in this work. (b) Details on the ECH power high duty cycle modulation (P3 in figure). (c) $\mathrm{ECH}$ power density deposition profiles, comparing the cases with $\mathrm{ECH}$ on- versus off- axis (discharges \#59113 and \#59112/59111, respectively). P3 phase is refereed to as 'NBI+ECH', while P5 is simply called 'ECH'. (d) NBI power density delivered to electrons. P1 is referred to as 'NBI' and it corresponds to discharge \#59113.

tained imposing $I_{\text {inductive }}=I_{p}-I_{\mathrm{cd}}-I_{\mathrm{bs}}$ with non-inductive current from ASTRA NBICD interpretative results and TorayGA [22] (ECCD) and the neoclassical conductivity and bootstrap current from Sauter's formulas [23] and kinetic data. We have checked that further interpretative ASTRA runs match the experimental plasma loop voltage. Constant radial profiles are assumed for $Z_{\text {eff }}$, with values ranging from 2.25 to 2.8 for the considered cases (see Table I). Fast ions (FI) are produced by NBI. Since we were interested in evaluating the FI effect on microturbulence, the FI density and temperature have been estimated in the phases with NBI injection of interest for GK investigation (NBI/NBI+ECH on-axis, which correspond to $\mathrm{P} 1 / \mathrm{P} 3$ phases of discharge \#59113, respectively), by means of interpretative ASTRA runs using the ASTRA NBI module, which assumes FI Neoclassical transport. The 
FI density and temperature profiles are shown in Fig.2 (a) and (b) respectively, compared with the corresponding electron quantities. FI have been modelled for simplicity by assuming isotropic (Maxwellian) velocity distributions. The associated FI temperature has been accordingly computed as $T_{\mathrm{FI}}=0.5 *\left(p_{\|, \mathrm{FI}}+p_{\perp, \mathrm{FI}}\right) / n_{\mathrm{FI}}$, where $p_{\|, \mathrm{FI}}, p_{\perp, \mathrm{FI}}$ and $n_{\mathrm{FI}}$ are the longitudinal pressure, perpendicular pressure and density of FI. The investigation of the impact of non-Maxwellian anisotropies is beyond the scope of this work.

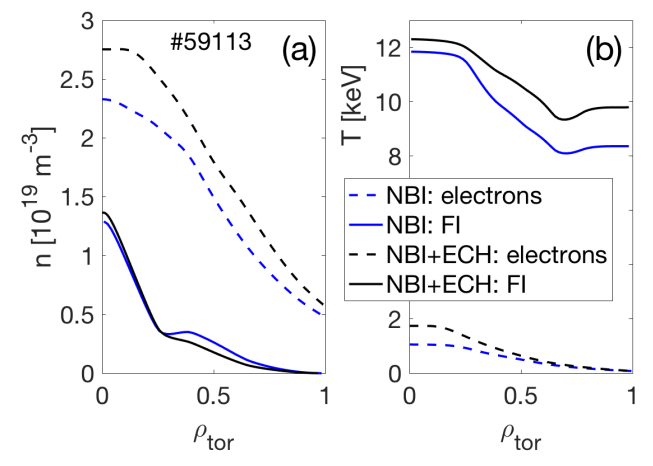

Figure 2. (color online) FI density (a) and temperature (b) profiles, for the considered phases with NBI (ECH on-axis, \#59113), compared with electron corresponding quantities.

When FI are considered, the ion density has been adapted in order to satisfy neutrality $\left(n_{i}=n_{e}-6 n_{c}-n_{F I}\right)$. NBI also drive toroidal rotation, which results in a finite $E \times B$ shearing rate, that could stabilise turbulence. The $E \times B$ shearing has been taken into account in the NL GK simulations of NBI phases, and it has been estimated from the measured background carbon toroidal velocity. The corresponding toroidal angular velocity profiles, measured with CXRS, are shown in Fig.3 for the cases with $\mathrm{ECH}$ on-axis. The $E \times B$ shearing effect has been neglected for the pure ECH case, due to the much smaller intrinsic rotation compared with the NBI-driven one (see red curve in Fig.3). This calculation assumes the plasma flow to be purely toroidal and identical for all species (which is the limit obtained for $\rho^{\star}=\rho_{s} / a \rightarrow 0$ for neoclassical poloidal rotation, where $\rho_{s}=c_{s} / \Omega_{i}$ is the sound Larmor radius and $a$ is the minor radius of the plasma, with $c_{s} \equiv \sqrt{T_{e} / m_{i}}$ the ion sound speed and $\Omega_{i}$ the ion cyclotron frequency) [24]. Retaining a finite neoclassical poloidal rotation (i.e. accounting for finite $\rho^{\star}$ ) would modify $\gamma_{E}$ by some $20-30 \%$.

Finally, the magnetic equilibrium is computed using the ideal MHD solver CHEASE [25], whose output is directly interfaced to GENE. The magnetic equilibrium and main plasma parameters for the three considered phases of the discharge with ECH deposition on-axis are shown in Fig.4. Figure 4 (a) shows the poloidal cross section of the magnetic surfaces, with the three phases represented by different colours. The safety factor radial profiles are shown in Fig.4 (b), while electron density and electron/ion temperature profiles are illustrated in Fig.4 (c) and (d)/(e), respectively. A $T_{i}$ flattening is observed when ECH is injected in presence of NBI, as can be seen comparing the blue and black lines in Fig.4 (e). This could be due to the different period of the ST in the two phases

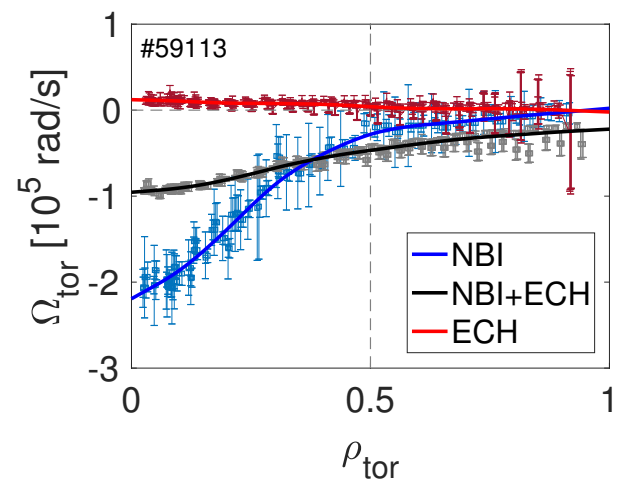

Figure 3. (color online) Carbon toroidal angular velocity profiles for the considered phases with NBI (ECH on-axis, \#59113). $\Omega_{\text {tor }}$ sign follows TCV conventions, i.e. $\Omega_{\text {tor }}>0$ corresponds to counterclockwise rotation as seen from above the tokamak. The plasma current $I_{p}$ is clockwise, therefore the toroidal rotation is co-current when NBI is injected, since $\Omega_{\text {tor }}<0$.

of the discharge. Indeed, the sawtooth period is more than halved for the mixed NBI/ECH case compared with the pure NBI one ( $4 \mathrm{~ms}$ compared with $10 \mathrm{~ms}$ ), therefore the $T_{i}$ profile does not have sufficient time to relax between sawtooth crashes for the mixed NBI/ECH case, resulting in a flattening.

Figures 5 (a) and (b) show the electron temperature variation with varying ECH deposition radius from on-axis to offaxis in the mixed NBI/ECH and ECH cases respectively.

The mid-radius position $\rho_{\text {tor }}=0.5$ is considered in the present analysis (where not stated differently), since it is the outer possible radius that allows on- vs off-axis ECH scan, due to mechanical constraints of the TCV ECH system. As a consequence, at that radius it is possible to have similar $T_{e}$ with different $\left|\nabla T_{e}\right|$ (see Fig.5 (a)/(b)). The main experimental parameters of interest at $\rho_{\text {tor }}=0.5$ are summarised in Table I. The normalised radial logarithmic gradients of the $f$ profiles $(f=n, T)$ are here defined as $R / L_{f}=-(R / \alpha) d \ln f / d \rho_{\text {tor }}$, where $\alpha=\sqrt{\Phi_{\text {edge }} / \pi B_{0}} \simeq$ $30 \mathrm{~cm}$ is a measure of average minor radius on LCFS. The other parameters are the safety factor $q$, the magnetic shear $\hat{s}=d \ln q / d \ln \rho_{\text {tor }}$, the ratio of the plasma pressure to the magnetic pressure $\beta=2 \mu_{0} n_{e} T_{e} / B_{0}$, with $\mu_{0}$ the vacuum permeability. Since the electron-ion collision rate depends on $n_{i}$, which is modified for simulations with FI $\left(n_{i}\right.$ is adapted using neutrality), the GENE collision parameter $v_{c}=2.3031 \times 10^{-5} \ln \Lambda R[m] n_{e}\left[10^{19} m^{-3}\right] / T_{e}[k e V]^{2}$ is listed in its place, since it only depends on the measured quantities $n_{e}, T_{e}$. Here, $\ln \Lambda=24-\ln \left(\sqrt{10^{13} n_{e}\left[10^{19} m^{-3}\right]} / 10^{3} T_{e}[k e V]\right)$ is the Coulomb logarithm. The electron-ion thermal collision rate can be easily evaluated for each case as $v_{e i}=$ $4\left(n_{i} / n_{e}\right) \sqrt{T_{e} / m_{e}} v_{c} / R$. Finally, the $E \times B$ shearing rate $\gamma_{E}=$ $\left(\rho_{\text {tor }} / q\right) \partial \Omega_{\text {tor }} / \partial \rho_{\text {tor }}$ is provided, where $\Omega_{\text {tor }}$ is the carbon toroidal angular velocity $\left(\Omega_{\text {tor }}\right.$ sign follows TCV conventions, see Fig.3). The parallel flow shear $\gamma_{p}$ was computed consistently with the pure toroidal flow assumption $\left[\gamma_{p} \simeq(q / \epsilon) \gamma_{E}\right.$, where $\epsilon=0.5 \alpha / R$ is the inverse aspect ratio at mid-radius].

The GENE code adopts a field-aligned coordinate system $(x, y, z)$ in configuration space, while $\left(\mathrm{v}_{\|}, \mu\right)$ are used as veloc- 

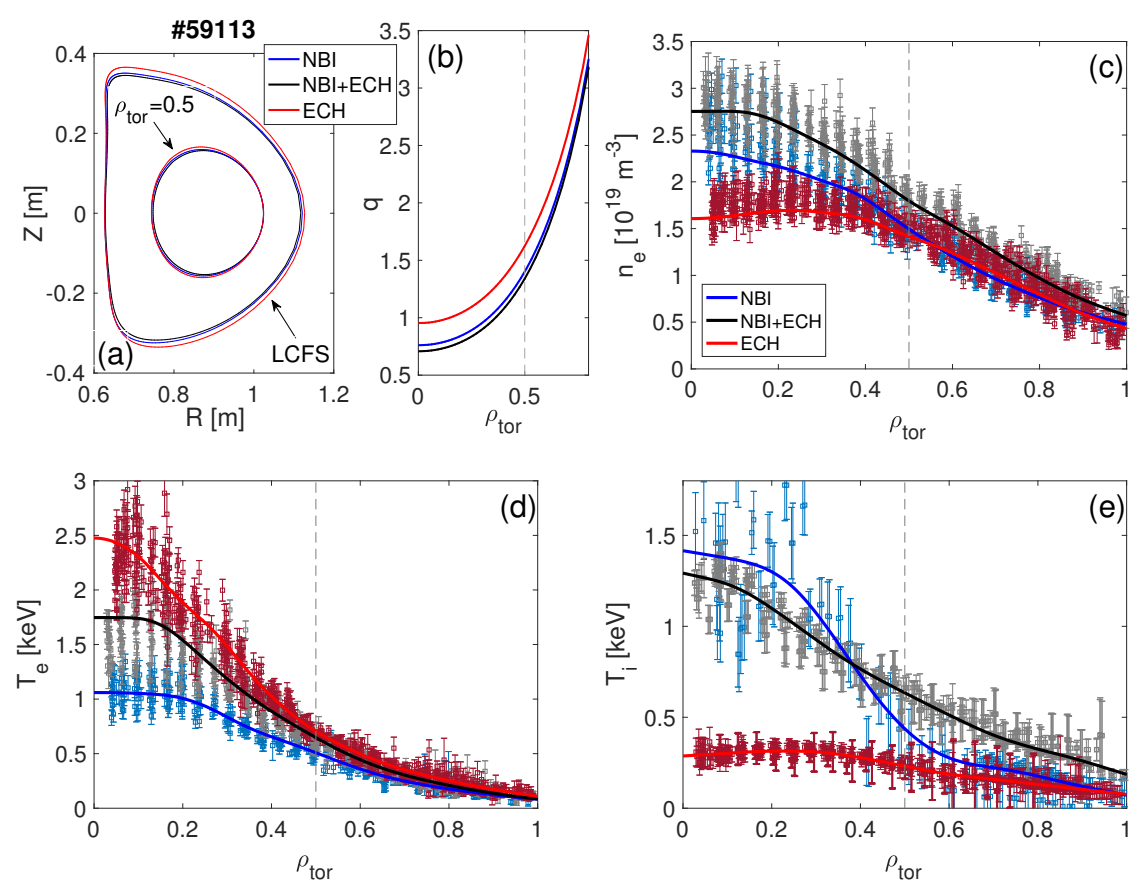

Figure 4. (color online) Magnetic equilibrium and main plasma parameters for the three cases of interest for the GK analysis (ECH on-axis, discharge \#59113). (a) CHEASE reconstruction of the poloidal cross section of the magnetic equilibrium. Magnetic surfaces corresponding to the three cases are shown in different colours. (b) Safety factor profiles. (c), (d) and (e) electron density, electron temperature and ion temperature profiles, respectively.
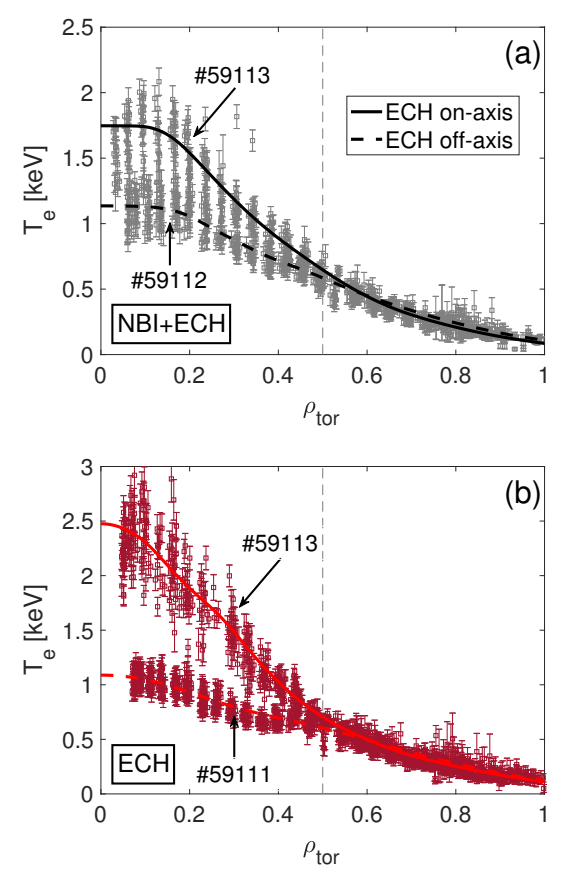

Figure 5. (a) and (b) Electron temperature profiles, comparing ECH on- versus off- axis deposition, for the NBI+ECH and ECH cases respectively.

ity variables in the reduced 2-dimensional GK velocity space. Here $(x, y, z)$ represent the radial, the binormal and the parallel positions respectively $(x=$ const $\& y=$ const define a magnetic field line, while $z$ sets the position along that line), $\mu=m \mathrm{v}_{\perp}^{2} / 2 B$ is the magnetic moment and $\mathrm{v}_{\|}$is the parallel velocity. In the flux-tube version of the code, Fourier representation is used for both the $x$ and $y$ directions. A typical grid size for a linear flux-tube simulation with fixed mode number $k_{y}$ with respect to the $y$ direction $\left(k_{y}=n q / \alpha \rho_{\text {tor }}\right.$, where $n$ is the toroidal mode number) is $n_{k x} \times n_{z} \times n_{\mathrm{v} \|} \times n_{\mu}=$ $48 \times 32 \times 64 \times 16$, while a typical NL simulation grid size is $n_{k x} \times n_{k y} \times n_{z} \times n_{\mathrm{v} \|} \times n_{\mu}=256 \times 64 \times 32 \times 64 \times 16$. To collect sufficient statistics, the NL flux-tube simulations have been run in time up to at least $t_{\max } c_{s} / R \sim 100$, or up to $t_{\max } c_{s} / R \sim 300$ when necessary. Convergence tests have been performed to check the reliability of the results. In all the simulations, electrons, carbon and FI (when present) have been treated as a self-consistent gyrokinetic species.

\section{EXPERIMENTAL ANALYSIS}

\section{A. Steady state scan}

In order to estimate the stiffness of the electron heat transport, the experimental heat flux densities in gyro-Bohm units $q_{e}[\mathrm{~GB}]$ are shown in Fig.6 (a) versus $R / L_{T e}$, comparing the two phases $\mathrm{NBI}+\mathrm{ECH} / \mathrm{ECH}$ (P3/P5) of the two discharges with ECH deposition on- and off- axis (\#59113 and \#59112, respectively), adding the NBI (P1, \#59113) data for completeness, in view of the following GK analysis where $\mathrm{NBI} / \mathrm{NBI}+\mathrm{ECH} / \mathrm{ECH}$ cases are compared. Here, $q_{e}[\mathrm{~GB}]=$ $q_{e} / q_{\mathrm{GB}}$, where $q_{e}$ is the radial heat flux per unit surface (the 


\begin{tabular}{|c|c|c|c|c|c|}
\hline & & \multicolumn{2}{|c|}{ ECH on-axis } & \multicolumn{2}{|c|}{ ECH off-axis } \\
\hline & NBI & $\mathrm{NBI}+\mathrm{ECH}$ & $\mathrm{ECH}$ & $\mathrm{NBI}+\mathrm{ECH}$ & $\mathrm{ECH}$ \\
\hline Discharge $n^{\circ}$ & \multicolumn{3}{|c|}{59113} & 59112 & 59111 \\
\hline$n_{e}\left[10^{19} \mathrm{~m}^{-3}\right]$ & 1.49 & 1.80 & 1.42 & 1.73 & 1.21 \\
\hline$n_{F I}\left[10^{19} \mathrm{~m}^{-3}\right]$ & 0.25 & 0.18 & & & \\
\hline$Z_{\text {eff }}$ & 2.80 & 2.80 & 2.50 & 2.70 & 2.25 \\
\hline$T_{e}[\mathrm{keV}]$ & 0.51 & 0.65 & 0.70 & 0.55 & 0.58 \\
\hline$T_{i}[\mathrm{keV}]$ & 0.44 & 0.63 & 0.23 & 0.51 & 0.19 \\
\hline$T_{\mathrm{FI}}[\mathrm{keV}]$ & 9.24 & 10.57 & & & \\
\hline$T_{e} / T_{i}$ & 1.16 & 1.03 & 3.04 & 1.08 & 3.05 \\
\hline$\tau$ & 3.25 & 2.88 & 7.6 & 2.92 & 6.86 \\
\hline$R / L_{n e}$ & 6.07 & 4.85 & 3.69 & 4.85 & 3.89 \\
\hline$R / L_{n F I}$ & 10.80 & 13.71 & & & \\
\hline$R / L_{T e}$ & 8.71 & 10.20 & 10.59 & 7.36 & 5.48 \\
\hline$R / L_{T i}$ & 15.07 & 5.55 & 5.94 & 8.51 & 3.68 \\
\hline$R / L_{T F I}$ & 2.29 & 1.67 & & & \\
\hline$q$ & 1.42 & 1.34 & 1.65 & 1.46 & 2.03 \\
\hline$\hat{s}$ & 1.14 & 1.19 & 0.99 & 1.09 & 0.78 \\
\hline$\beta\left[10^{-3}\right]$ & 1.54 & 2.37 & 2.01 & 1.93 & 1.42 \\
\hline$v_{c}\left[10^{-3}\right]$ & 1.77 & 1.33 & 0.91 & 1.77 & 1.13 \\
\hline$\gamma_{E}\left[c_{s} / R\right]$ & 0.34 & 0.14 & & & \\
\hline
\end{tabular}

Table I. Main plasma parameters at $\rho_{\text {tor }}=0.5$. FI parameters are only shown for the cases with ECH deposition on-axis, of interest for the GK analysis.

magnetic surface area is $\sigma \simeq 5.3 \mathrm{~m}^{2}$ for all the considered cases, see Fig.4 (a)) and $q_{\mathrm{GB}}=\sqrt{m_{i}} n_{e} T_{e}^{5 / 2} / e^{2} R^{2} B_{0}^{2}$ is the gyro-Bohm normalisation. $q_{e}$ is computed by volume integration of $P_{\mathrm{TOT}}=P_{\mathrm{ECH}}+P_{\mathrm{NBI}}+P_{\mathrm{OHM}}-P_{\mathrm{EI}}$ up to $\rho_{\text {tor }}=0.5$, where $P_{\mathrm{ECH}}, P_{\mathrm{NBI}}, P_{\mathrm{OHM}}$ and $P_{\mathrm{EI}}$ are the power densities corresponding to ECH, NBI power transferred to electrons, ohmic heating and electron/ion power exchange, respectively. While $P_{\mathrm{NBI}}, P_{\mathrm{OHM}}$ and $P_{\mathrm{EI}}$ are obtained from ASTRA interpretative runs, $P_{\mathrm{ECH}}$ is evaluated assuming a gaussian profile, with location, amplitude and width that are optimised in order to match the amplitudes and phases of the ECH modulation, using ASTRA (see Section IV). The resulting $P_{\mathrm{ECH}}$ has been compared with the output of the ray tracing code TORAY-GA, to check consistency. The convective contribution $(5 / 2) T_{e} \Gamma_{e, N B I}$ to $q_{e}$ coming from the NBI particle source, where $\Gamma_{e, N B I}$ is the electron particle flux due to the NBI source, has been neglected since it is a small correction (it contributes $\sim 5 \%$ for the $\mathrm{NBI}+\mathrm{ECH}$ on-axis case). The error bars on $R / L_{T e}$ are estimated to be $\pm 20 \%$, given that the error bars on density and temperature are approximately $\pm 10 \%$ around the radius of analysis. The error bars on $q_{e}[\mathrm{~GB}]$ are obtained considering a $\pm 5 \%$ error bar on $q_{e}$ (mainly due to $\mathrm{ECH}$ power uncertainty) and furthermore propagating the $n_{e}, T_{e}$ errors through $q_{\mathrm{GB}}$, resulting in an effective relative error of $\pm 30 \%$. Moreover, an analytic estimate of the linear ETG thresholds corresponding to the experimental conditions has been added to Fig.6, ac- cording to [18]:

$$
\begin{aligned}
{\left[\frac{R}{L_{T e}}\right]_{\mathrm{TH}, \mathrm{ETG}} \simeq \xi \max } & \left\{(1+\tau)\left(1.33+1.91 \frac{\hat{s}}{q}\right)(1-1.5 \xi \epsilon) \times\right. \\
& \left.\times\left(1+0.3 \epsilon \frac{d \kappa}{d \epsilon}\right), 0.8 \frac{R}{L_{n}}\right\}
\end{aligned}
$$

where $\kappa$ is the elongation and $\xi=a / \alpha$, with $a \simeq 25 \mathrm{~cm}$ the plasma geometrical minor radius at the midplane, is introduced to account for the fact that the estimate in [18] is obtained defining $L_{T e}$ using $r$ (geometrical minor radius at the midplane) instead of $\rho_{\text {tor }}$. This estimate of the linear ETG threshold has been validated for our case by means of linear GK simulations (see Section IV A). Error bars on $\left[R / L_{T e}\right]_{\mathrm{TH}}$ are provided as well, propagating the errors on $\hat{s} / q, \epsilon, d \kappa / d \rho_{\text {tor }}$ $( \pm 10 \%)$ and $\tau\left( \pm 20 \%\right.$, obtained propagating the errors on $Z_{\text {eff }}$, $T_{e}, T_{i}$, assuming $\pm 10 \%$ error bars).

The ECH (P5) fluxes show moderate stiffness (see the red dashed line), consistent with TEM driven transport. This picture is consistent with the fact that both on- and off-axis cases are below the corresponding linear ETG thresholds. For the mixed NBI/ECH (P3) cases it is not possible to accurately evaluate the stiffness, since the $R / L_{T e}$ error bars corresponding to the case with ECH on- and off-axis overlap, implying a large uncertainty on the slope of $q_{e}[\mathrm{~GB}]$ vs $R / L_{T e}$. In order to overcome this issue, a perturbative analysis of the propagation of the heat wave produced by the $\mathrm{ECH}$ modulation has been performed (see section III B), allowing to estimate the stiffness and threshold for those cases. It is worth noting that the mixed NBI/ECH cases are close to/above the linear ETG threshold, thus being good candidates to test the contribution of the ETGs to transport at mid-radius. Finally, the NBI (P1) case is also close to threshold. It is important to note that other parameters than $R / L_{T e}$ vary between on- and off-axis cases, therefore Fig.6 (a) does not show the 'pure' $R / L_{T e}$ dependence of the electron heat flux. For example, it is worth pointing out that while the linear ETG thresholds for the mixed NBI/ECH cases with ECH deposition on- vs offaxis are similar to each other, which is expected since $T_{e}$ and $Z_{\text {eff }}$ are similar (see Fig.5 and Table I), the ECH cases have quite different thresholds. This is principally due to the different $q$ profiles, leading to $\hat{s} / q$ values that are $\sim 50 \%$ different in the two cases. Finally, the different contributions to $q_{e}$ are detailed in Fig.6 (b), showing that the ECH component is dominant when present, while NBI and ohmic contributions are comparable when NBI is present. The electron-ion heat exchange is always a small correction. It is worth noting that there is no contribution from $\mathrm{ECH}$ for the $\mathrm{NBI}+\mathrm{ECH}$ off-axis case, since the ECH is entirely deposited outside $\rho_{\text {tor }}=0.5$ (see Fig.1 (c)).

\section{B. perturbative analysis}

A perturbative approach has been followed, in order to estimate the threshold and stiffness of the electron heat transport where it could not be unequivocally computed with the steady state scan (see section III A), i.e. for the mixed NBI/ECH 

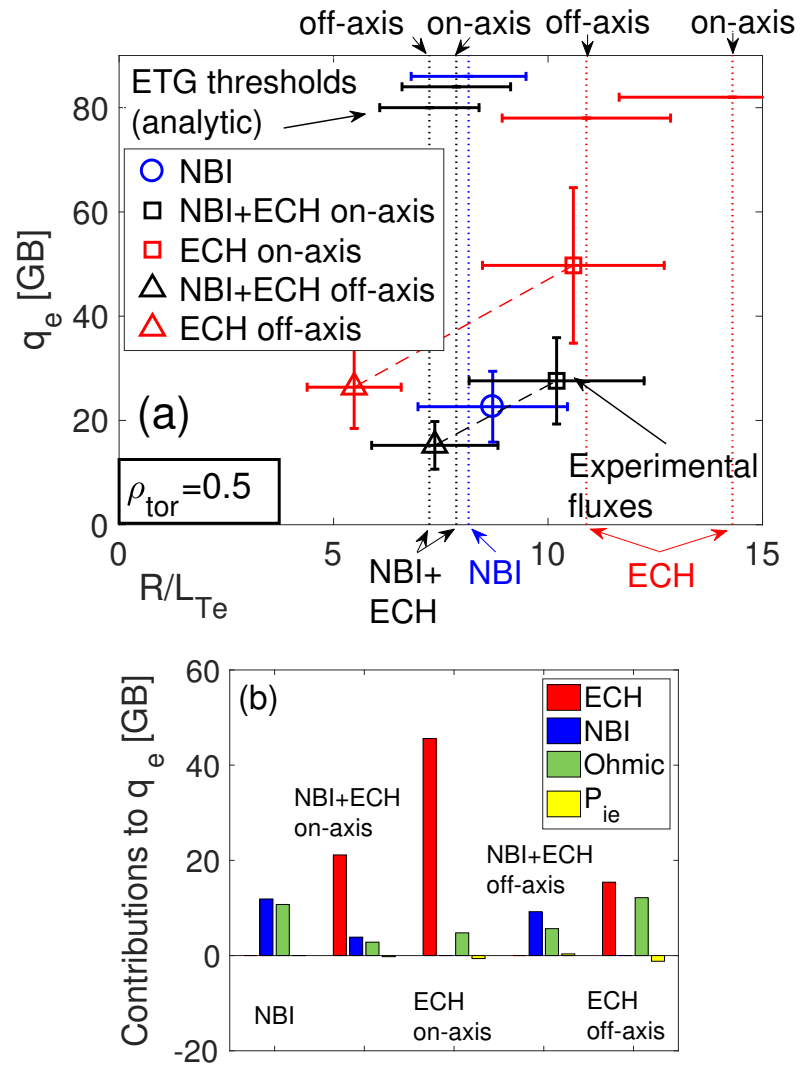

Figure 6. (color online) (a) Experimental electron heat flux density at $\rho_{\text {tor }}=0.5$ in gyro-Bohm units $q_{e}[\mathrm{~GB}]$ versus $R / L_{T e}$, compared with analytic ETG linear thresholds $\left[R / L_{T e}\right]_{\mathrm{TH}}$ (vertical dotted lines; cases with ECH on-/off- axis are indicated with 'on-axis'/'off-axis', respectively); (b) Different contributions to $q_{e}[\mathrm{~GB}]$ for the five cases considered in (a).

cases, as well to verify the results that have been obtained for the pure ECH ones. The analysis of the heat pulse propagation due to either ECH modulation or ST activity has been performed with this aim. For the ECH modulation analysis, threshold and stiffness can be extracted from the knowledge of the amplitudes of the fluctuations $\delta T_{e}$ of the electron temperature due to the heat pulse and the phases between the first harmonics of the $\mathrm{ECH}$ power signal and the local $\delta T_{e}$ signals along the plasma radius. For the ST propagation analysis, the phases between the first harmonics of the $\delta T_{e}$ fluctuation at a fixed position close to the inversion radius and the $\delta T_{e}$ at outer radii are considered. The so obtained radial profiles of $\delta T_{e}$ amplitude and phases are matched with interpretative runs of a transport code, estimating the threshold and stiffness by allowing a suitable model for the electron heat flux.

Interpretative simulations have been performed with ASTRA, adopting an empirical critical gradient model (CGM) [26] for the evaluation of $q_{e}$. This features an electron heat flux of the form

$$
q_{e}=q_{e}^{\mathrm{res}}+q^{3 / 2} \chi_{s} \frac{n_{e} T_{e}^{2} \rho_{s}}{e B_{0} R^{2}} \frac{R}{L_{T e}}\left(\frac{R}{L_{T e}}-\kappa_{c}\right) \theta\left(\frac{R}{L_{T e}}-\kappa_{c}\right),
$$

where $q_{e}^{\text {res }}$ is a residual flux not driven by $R / L_{T e}$ (in particu- lar also accounting for ITG modes), $\kappa_{c}$ is the critical $R / L_{T e}$ value, $\chi_{s}$ is the stiffness coefficient and $\theta$ is the Heaviside function. In order to reproduce the data, two terms for the turbulent part have been considered in the form of Eq.2, corresponding to TEMs and ETGs, each with its threshold value and stiffness level, and then they have been added to obtain the total $q_{e}$. All values are empirically adjusted to fit the data, but an analytical functional form has been adopted at each radius for TEM and ETG thresholds. For the TEM term, $\kappa_{c}$ has been set proportional to A. Peeters linear estimate [27], i.e. $\kappa_{c}=\delta_{\mathrm{TEM}}\left[R / L_{T e}\right]_{\mathrm{TH}}$, TEM with:

$$
\begin{aligned}
{\left[\frac{R}{L_{T e}}\right]_{\mathrm{TH}, \mathrm{TEM}} } & =\xi \frac{0.357 \sqrt{\xi \epsilon}+0.271}{\sqrt{\xi \epsilon}}[4.90+ \\
& \left.-1.31 \frac{R}{L_{n}}+2.68 \hat{s}+\ln \left(1+20 v_{\mathrm{eff}}\right)\right],
\end{aligned}
$$

where $v_{\mathrm{eff}} \simeq n_{e}\left[10^{19} \mathrm{~m}^{-3}\right] Z_{\mathrm{eff}} / T_{e}[\mathrm{keV}]$ is the effective collisionality, while for the ETG term, it has been set proportional to F. Jenko linear estimate [18] (i.e. $\kappa_{c}=\delta_{\mathrm{ETG}}\left[R / L_{T e}\right]_{\mathrm{TH}}$, ETG with $\left[R / L_{T e}\right]_{\mathrm{TH}}$, ETG from Eq.1). The two adjustable factors $\delta_{\mathrm{TEM}}$ and $\delta_{\mathrm{ETG}}$, of order unity, allow for discrepancies between the linear thresholds and the actual (NL) ones. Polynomially increasing radial profiles have been considered for $\chi_{s}$. The values of $\delta_{\text {TEM,ETG }}$ and $\chi_{s}$ have been adjusted in order to consistently match the experimental $T_{e}$ and both the profiles of the amplitude and phase of the $T_{e}$ fluctuations with respect to the input $\mathrm{ECH}$ power signal. A localised large electron heat diffusivity $\chi \sim 10 \mathrm{~m}^{2} / \mathrm{s}$ in the region $\rho_{\text {tor }}<0.2$ has been assumed for the cases with ECH deposition on-axis, following the common practice, in order to keep $T_{e}$ close to the experimental profile despite the large ECH power density deposition in a region where turbulence is supposed to be suppressed. In order to have sufficiently time-resolved $T_{e}$ measurements, the ECE/CECE (Electron Cyclotron Emission diagnostic/Correlation ECE) data have been considered for this purpose instead of the Thomson ones. ECE data have been complemented with CECE ones at the smaller radii where the ECE channels were polluted by the pick-up from $\mathrm{ECH}$ power. ECE and CECE diagnostics use a different set of amplifiers and filters. The intermediate frequency (IF) amplifiers of the standard ECE system were saturated by the ECH pickup and caused the pollution in the signals, while the CECE IF amplifiers did not suffer from this. The comparison of ASTRA simulations and experimental phases and amplitudes is shown in Fig. 7. Only the phases of the first two harmonics are shown, since the $T_{e}$ signal is noisy for the third ones, due to the small amplitudes. The amplitude of the third harmonic was also not considered for the NBI+ECH on-axis case, because of ST pollution. A good agreement between ASTRA and experiment is obtained for all the cases except the mixed $\mathrm{NBI} / \mathrm{ECH}$ with ECH on-axis, where only the phases are well matched, while the amplitudes are overestimated by ASTRA. This can be explained looking at the corresponding $T_{e}$ signal. The $T_{e}$ time traces corresponding to the NBI+ECH on-axis case at $\rho_{\text {tor }}=0.1$ (close to ECH deposition) are shown in Fig. 8 (a), compared with ECH power signal. It is clear that large ST pollute the signal, interfering with the effect from the power 
modulation. This is even clearer looking at the corresponding amplitude spectra, comparing the ECH on-axis case with the $\mathrm{NBI}+\mathrm{ECH}$ on-axis one (see Fig.8 (b)). While in the ECH case the spectrum is clean and the corresponding $\mathrm{ECH}$ harmonics are distinguishable, in the mixed $\mathrm{NBI} / \mathrm{ECH}$ one large ST peaks are present at frequencies that are close to the main harmonic, with amplitudes which are of the same order as the ECH peak. For these reasons in the ASTRA fits we have given priority to reproducing the phase profiles, accepting an overestimate of the amplitudes. In fact, reproducing both the phase and amplitude profiles is impossible to achieve given the experimental level of power modulation and the levels of transport necessary to reproduce the phase profiles. $q_{e}^{\text {res }}$ has been set to zero for the mixed $\mathrm{NBI} / \mathrm{ECH}$ cases, consistent with a strong ITG stabilisation by FI (due to NBI). This picture was confirmed in the GK analysis, where a strong FI stabilisation of the ion scales has been found for the mixed $\mathrm{NBI}+\mathrm{ECH}$ on-axis case (see Section IV).

The so computed threshold and stiffness coefficients $\kappa_{c}$ and $\chi_{s}$, evaluated at $\rho_{\text {tor }}=0.5$, have been substituted in the CGM expression for $q_{e}$ (Eq.2), to obtain an estimate of $q_{e}$ [GB] vs $R / L_{T e}$. The results are in Fig.9, where they are compared with the experimental values. This plot adds the missing information from the pure steady-state plot of Fig.6 (a). First of all, we note that in the pure ECH cases the TEM and ETG thresholds are rather far apart and a TEM branch is followed by a discontinuously stiffer ETG one with increasing $R / L_{T e}$. We note that the stiffness of the on-axis and off-axis cases in fact differ significantly, which can be ascribed to the different q profiles obtained when moving the dominant $\mathrm{ECH}$ power from on-axis to off-axis. As a result, the two steady state points corresponding to pure ECH heating cannot be considered as lying on the same stiffness curve, although the error on TEM stiffness made by connecting the two steady-state points is not large because the off-axis point lies close to threshold. Secondly, for the mixed NBI/ECH cases the TEM and ETG thresholds are similar, resulting in a mixed continuous TEM/ETG branch with a stiffness resulting from the combination of the two branches. The proportion of TEM/ETG contributions varies with radius, due to the different radial dependence of the $\chi_{s}$ profiles, so that the importance of ETGs is larger at outer radii. Moreover, a different proportion of TEM/ETG is considered for the mixed $\mathrm{NBI} / \mathrm{ECH}$ cases with $\mathrm{ECH}$ deposition on- and off-axis respectively, by varying their corresponding stiffness profiles. For the 'on-axis' case, we were guided by the information coming from GK modelling (see section IV), indicating that the transport at mid-radius should be dominantly ETG, with small TEM ion-scale contribution and negligible ITG. As a consequence, it was possible to fit the modulation data with a dominantly ETG scenario, with negligible TEM and $q_{e}^{\text {res }}=0$. The off-axis case, on the other hand, shows larger $R / L_{T i}$ (see Table I), and therefore ITG modes could be expected to at least partially mitigate ETGs non-linearly. Consistently, it has been possible to fit the modulation data allowing a larger TEM/ETG balance, with finite $q_{e}^{\text {res }}$. For all the considered cases, the ETG thresholds are in good agreement with the linear ones (see section III), i.e. the $\delta_{\mathrm{ETG}}$ factors differ from unity by less that $20 \%$, with the exception of the
$\mathrm{NBI}+\mathrm{ECH}$ off-axis case, where the ETG threshold underpredicts the linear one by $\sim 35 \%$, but it should be noted that the TEMs are so much dominant on ETGs at smaller radii that the error bar on the ASTRA evaluation of the ETG threshold for this case could be large.

In order to test the consistency of the ASTRA estimate of $\kappa_{c}$ and $\chi_{s}$ at mid-radius for the $\mathrm{NBI}+\mathrm{ECH}$ on-axis case, where ST pollute the $T_{e}$ modulation measurements, an independent estimate of the stiffness was obtained by studying the ST propagation. Given the phase $\phi$ and the amplitude $A$ of the $T_{e}$ fluctuation, where $\phi$ is taken with respect to the $T_{e}$ channel at $\rho_{\text {tor }} \sim 0.3$, just outside the inversion radius, corresponding to the first harmonic of the ST (frequency $f \sim 230 \mathrm{~Hz}$ ), the perturbative thermal diffusivity $\chi_{\text {pert }}=-\left(1 / n_{e}\right) \partial q_{e} / \partial\left|\nabla T_{e}\right|$ is estimated as [28]:

$$
\chi_{\mathrm{pert}}=-\frac{(3 / 4) \omega}{\phi^{\prime}\left(A^{\prime} / A+1 / 2 r-1 / 2 r_{n}\right)},
$$

where $\omega=2 \pi f, r=0.5 \alpha$ and $r_{n}=n_{e} /\left|\nabla n_{e}\right|$. The ST phase and amplitude profiles are shown in Fig.10. $\phi^{\prime}$ and $A^{\prime} / A$ have been computed at $\rho_{\text {tor }}=0.5$ by linearly interpolating $\phi$ and $\ln A$ around the selected radius (solid lines). The so resulting stiffness is easily computed and indicated by a magenta line in Fig.9. The result seems consistent with the modulation analysis. Unfortunately, it was not possible to estimate the stiffness for the NBI only case analysing the ST signal, since for that phase a large ballistic component hides the diffusive propagation.

Summing up, according to this analysis, the pure ECH cases at mid radius are both below the ETG threshold and above the TEM threshold. A consistent picture of moderate stiffness emerges from both the heat flux scans and the modulation analysis. For the mixed NBI/ECH cases, the heat flux scans cannot provide a reliable estimate of stiffness due to the proximity of the two points and their uncertainties, so that the perturbative analysis becomes essential. This indicates that the two NBI/ECH cases at mid radius are both above the TEM and ETG thresholds, which are close to each other. The stiffness of the on-axis case is larger than in the pure ECH case, due to the ETG contribution, whilst the off-axis case features a lower stiffness consistent with less ETG contribution. Finally, it is worth pointing out that even if only the mixed NBI/ECH cases are above ETG threshold at $\rho_{\text {tor }}=0.5$, the ASTRA simulations indicate that ETGs are unstable for both pure ECH and mixed NBI/ECH cases at larger radii, due to the fact that the ETG $R / L_{T e}$ threshold decreases with radius. This is shown for the cases with ECH deposition on-axis in Fig.11, where the ETG and TEM threshold profiles are superimposed to $R / L_{T e}$ ones. Moreover, $\chi_{\text {turb }}=\left(q_{e}-q_{e}^{\text {res }}\right) / n_{e}\left|\nabla T_{e}\right|$ is added to the figure, to show the radial dependence of the turbulent transport.

As a consequence, the analysis of Fig.9 has been repeated at the larger radius $\rho_{\text {tor }}=0.7$. The results (see Fig.12) show that at $\rho_{\text {tor }}=0.7$ all cases are above the ETG threshold. 

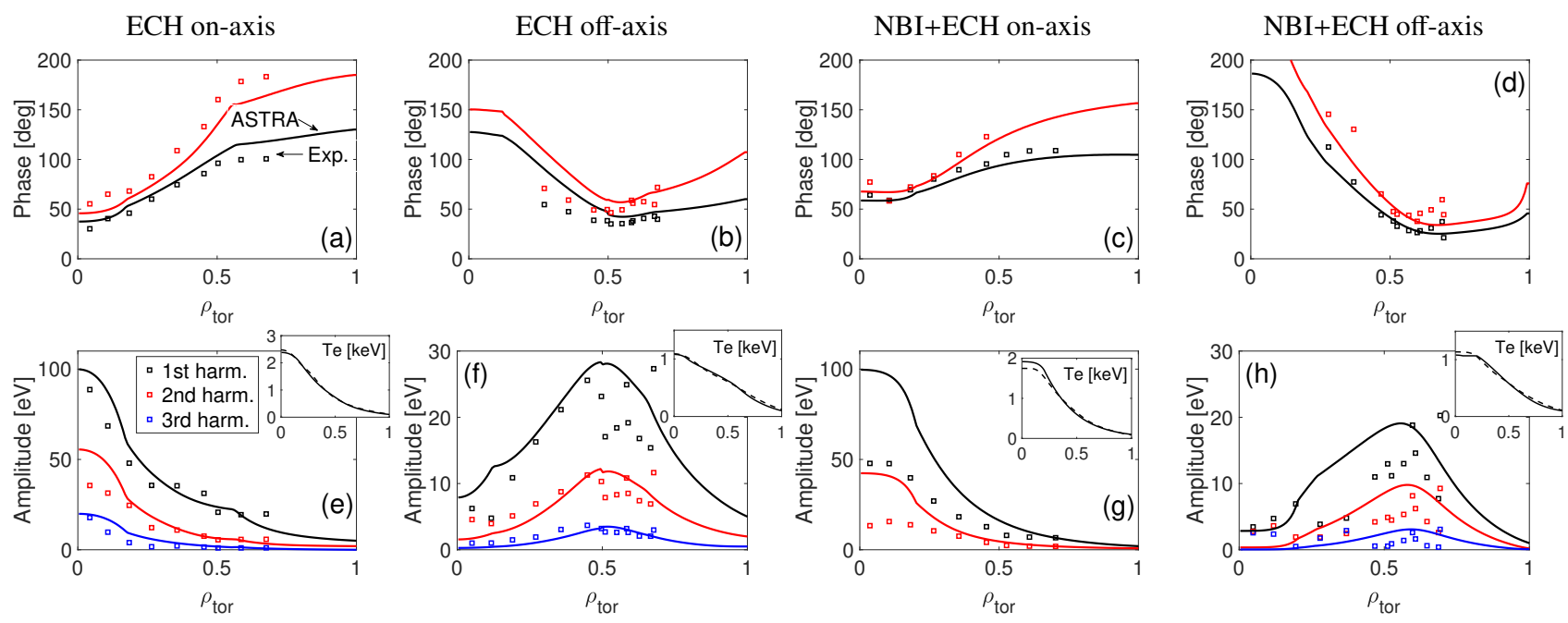

Figure 7. (color online) Comparison of the ASTRA simulations outputs (solid lines) with the experimental phases (a)-(d) and amplitudes (e)-(h) (square markers) of the first three harmonics of the ECH power signal. Only the phases of the first two harmonics are shown, since the third harmonic signal is always noisy due to small amplitude. Only the amplitudes of the first two harmonics are shown for the NBI+ECH on-axis case, since the third harmonic is completely hidden by the ST harmonics. The matching of the average $T_{e}$ is shown in the little boxes of (e)-(h) (ASTRA: solid; exp.: dashed). The four columns correspond to NBI+ECH on-axis, NBI+ECH off-axis, ECH on-axis and ECH off-axis cases, respectively.
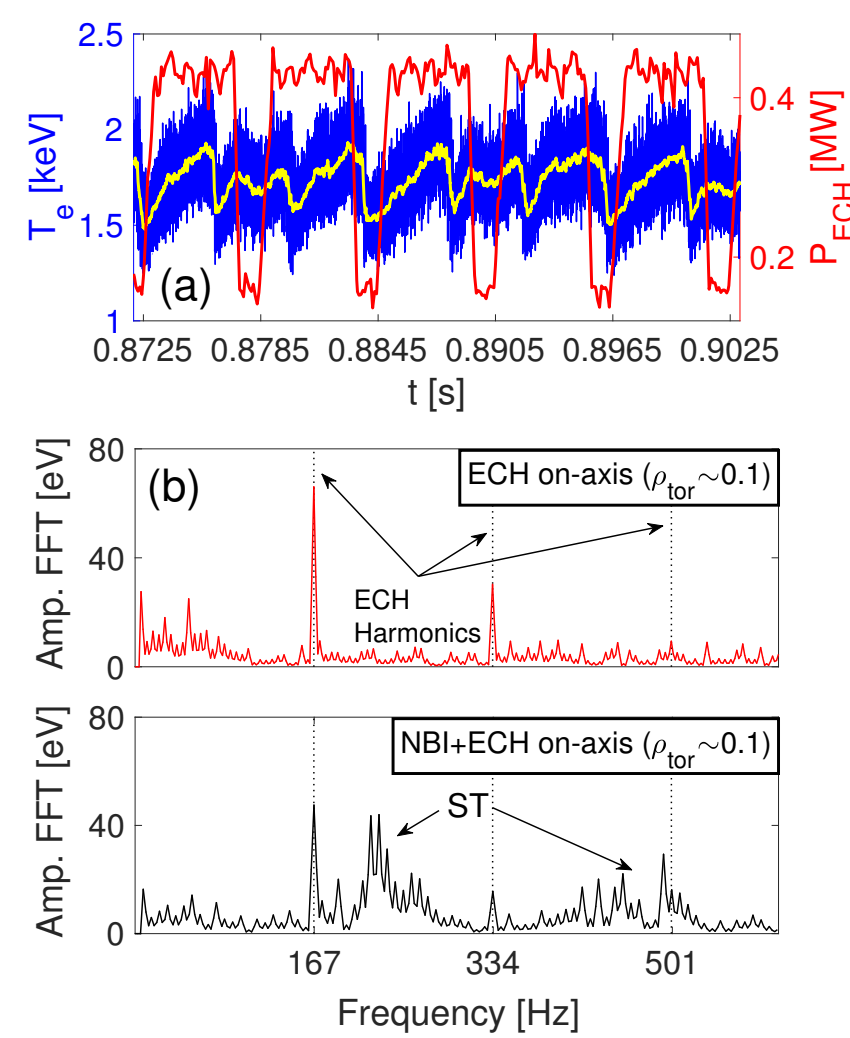

Figure 8. (color online) (a) Time slice of $T_{e}$ signal (CECE) for the $\mathrm{NBI}+\mathrm{ECH}$ on-axis case (blue), with $\mathrm{ECH}$ power signal superimposed (red). The yellow line is a smoothing of the $T_{e}$ signal (average over $10^{-4} \mathrm{~s}$ around each time); (b) FFT of the amplitude of the $T_{e}$ fluctuations for the CECE channel at $\rho_{\text {tor }} \sim 0.1$, close to ECH deposition.

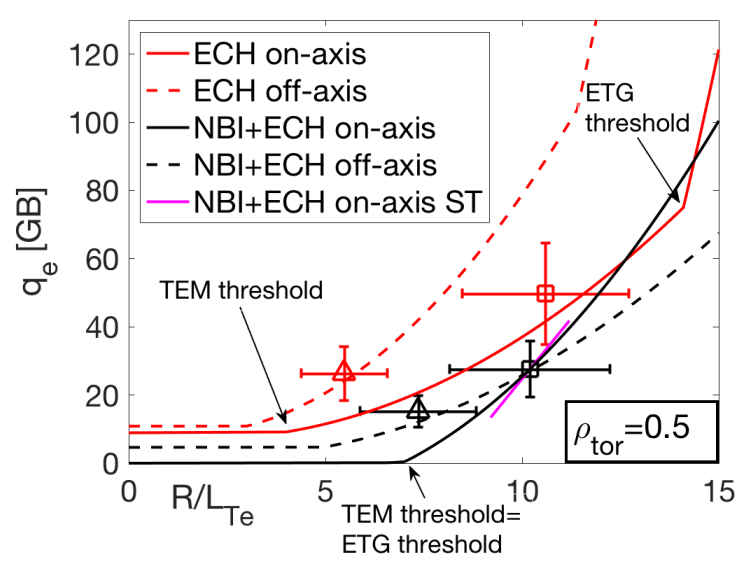

Figure 9. (color online) Comparison of the ASTRA electron heat flux in GB units $q_{e}[\mathrm{~GB}]$ versus $R / L_{T e}$ (solid/dashed lines for on/off-axis respectively) with the experimental values at $\rho_{\text {tor }}=0.5$. See Fig.6 for experimental points legend. The TEM and ETG thresholds are indicated for the cases with ECH on-axis. For further information on TEM and ETG thresholds, we refer to Fig11.

\section{GYROKINETIC MODELLING}

\section{A. linear results: multi-scale spectra and ETG thresholds}

In order to characterise the turbulence in the three selected phases (NBI, NBI+ECH on-axis, ECH on-axis), linear $k_{y}$ scans of flux-tube GK simulations have been performed with the GENE code at $\rho_{\text {tor }}=0.5$, with experimental mean parameters (see Table I, on-axis cases), computing the growth rate and frequency spectra. A reduced value $Z_{\text {eff }}=2$ of the effective charge has been considered given that only carbon im- 


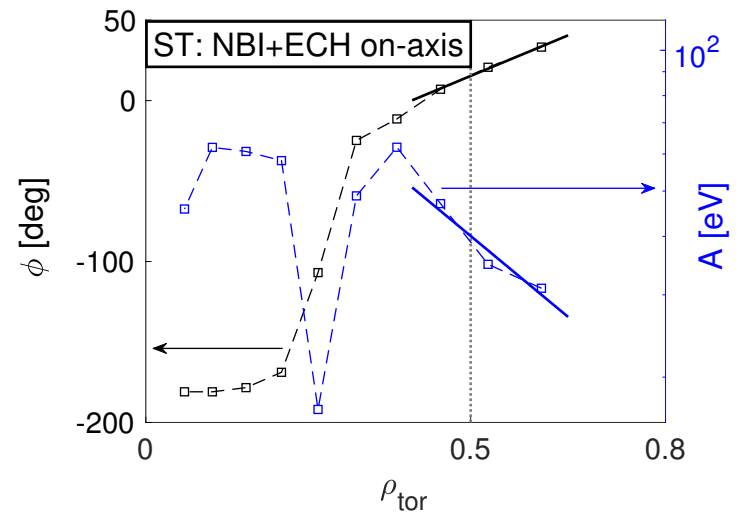

Figure 10. (color online) Phase $\phi$ (black) and amplitude $A$ (blue) of the $T_{e}$ fluctuation (ECE LFS) due to the first harmonic (frequency $f \sim 230 \mathrm{~Hz}$ ) of the ST propagation. The phase is computed with respect to the $T_{e}$ ECE HFS channel at $\rho_{\text {tor }} \sim 0.3$, just outside the inversion radius. The solid lines represent the linear fit of $\ln A$ around the radius of analysis $\rho_{\text {tor }}=0.5$.
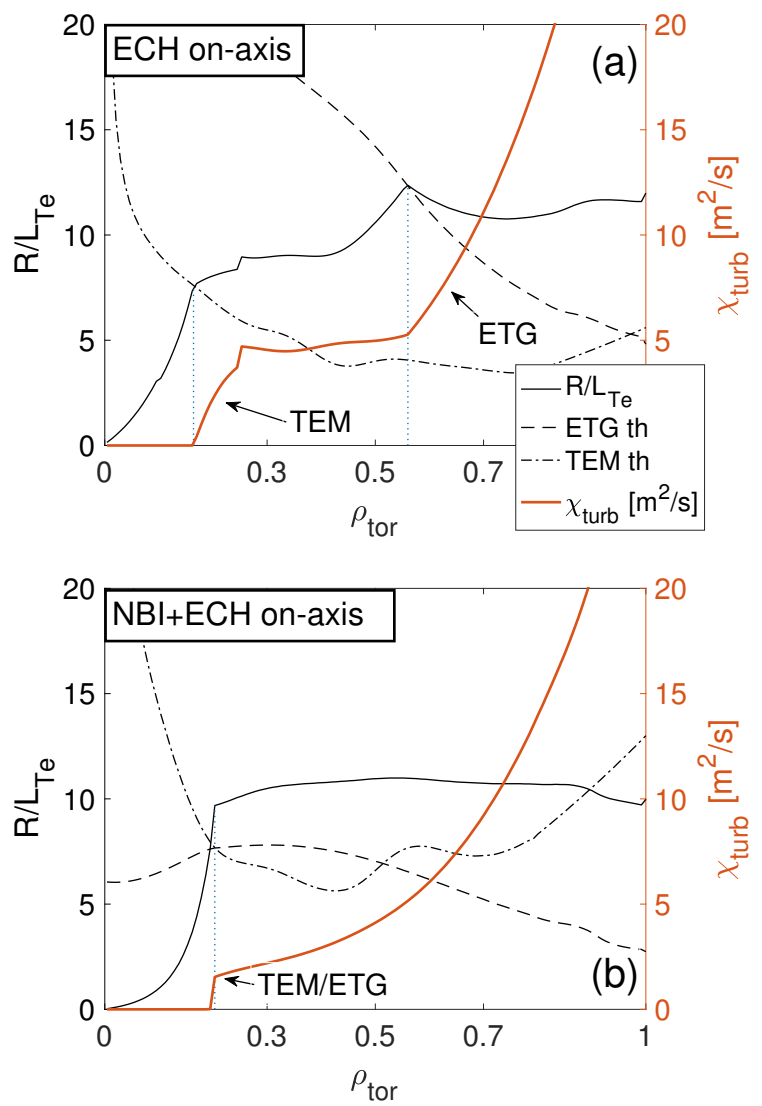

Figure 11. (color online) ECH deposition on-axis: ASTRA simulation output for $\mathrm{ECH}$ on-axis (a) and $\mathrm{NBI}+\mathrm{ECH}$ on-axis (b) cases respectively: comparison of $R / L_{T e}$ profile (solid) with TEM (dasheddotted) and ETG (dashed) thresholds, superimposed to $\chi_{\text {turb }}$ (orange).

purity has been accounted for in the simulations, corresponding to a $n_{c} / n_{e} \simeq 3 \%$ carbon/electron density ratio. The study

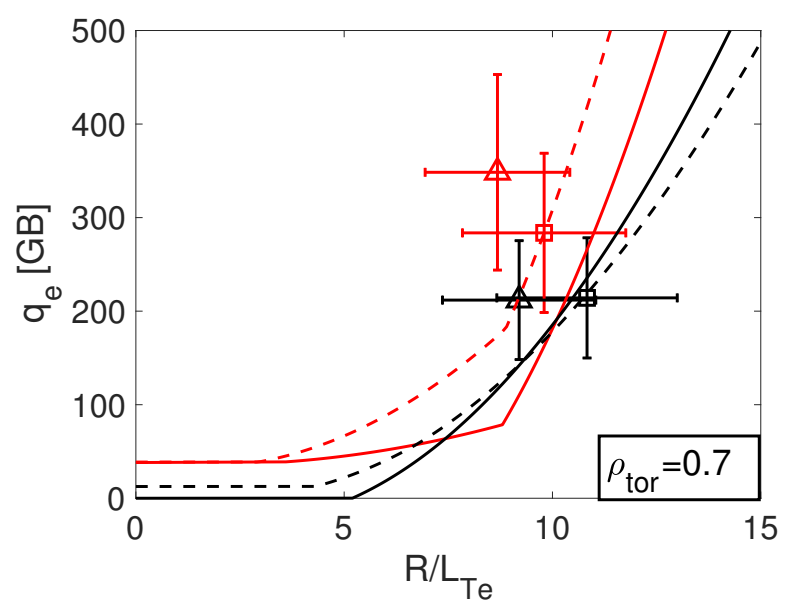

Figure 12. (color online) Comparison of the ASTRA electron heat flux in GB units $q_{e}$ [GB] versus $R / L_{T e}$ with the experimental values at $\rho_{\text {tor }}=0.7$. See Fig.6 for experimental points legend

of the effect of other impurities is beyond the scope of this work. The eigenvalues which have been obtained with and without adding FI have been compared to single out their effect. The results are shown in Fig.13. Figure 13 (a) displays the ratio of the growth rate $\gamma$ and the corresponding $k_{y}$, in order to test the importance of the electron scales in the heat transport, according to [29]. ETGs are expected to significantly contribute to the electron heat flux if the electron scale maximum $\left(\gamma / k_{y}\right)_{\text {e, } \max }$ is larger than the ion scale maximum $\left(\gamma / k_{y}\right)_{\mathrm{i}, \max }$. The only phase where ETG are expected to significantly impact the electron heat transport, according to this principle, is the mixed NBI/ECH one. In this case, NBI produces a decrease of the ETG $R / L_{T e}$ threshold, by increasing $T_{i} / T_{e}$, while $R / L_{T e}$ increases due to $\mathrm{ECH}$, leading to the optimal condition to observe ETGs. It is also clear that the condition $\left(\gamma / k_{y}\right)_{\mathrm{e}, \max } /\left(\gamma / k_{y}\right)_{\mathrm{i}, \max }>1$ is mainly obtained thanks to a strong FI stabilisation of ion scale turbulence (TEM). FI also have a small destabilising effect on ETGs in both NBI and mixed NBI/ECH phases. The effect of $E \times B$ shearing, due to NBI-driven rotation, on transport, can be foreseen by these preliminary linear results. The ratio of $\gamma_{E}$ (see Table I) to the maximum linear growth rate at the ion scale $\gamma_{\mathrm{i}, \max }$ is equal to $\gamma_{E} / \gamma_{\mathrm{i}, \max }=43 \% / 37 \%$ for the pure NBI cases with/without FI respectively, while it is equal to $\gamma_{E} / \gamma_{\mathrm{i}, \max }=80 \% / 29 \%$ for the mixed NBI/ECH cases with/without FI. These means that even if $\gamma_{E}$ is larger for the pure NBI case, the largest effect of $E \times B$ is expected in the mixed NBI/ECH case with FI, for the combined effect of the stronger FI stabilisation in the mixed $\mathrm{NBI} / \mathrm{ECH}$ case and the smaller growth rate compared with the pure NBI one. These results are confirmed by NL ones for the mixed NBI/ECH case in section IV B.

Looking at the mode frequencies (Fig. 13 (b)), the cases with ECH (both with and without FI) show TEM dominated ion scale turbulence, while the NBI only case is ITG dominated (due to high $R / L_{T i}$; see Table I and blue curve in Fig.4). It is worth stressing that the notation 'TEM'/'ITG' is used in this work to refer to modes with negative/positive frequency 
respectively at ion scales, while 'ETG' refers to modes with negative frequency at electron scales.
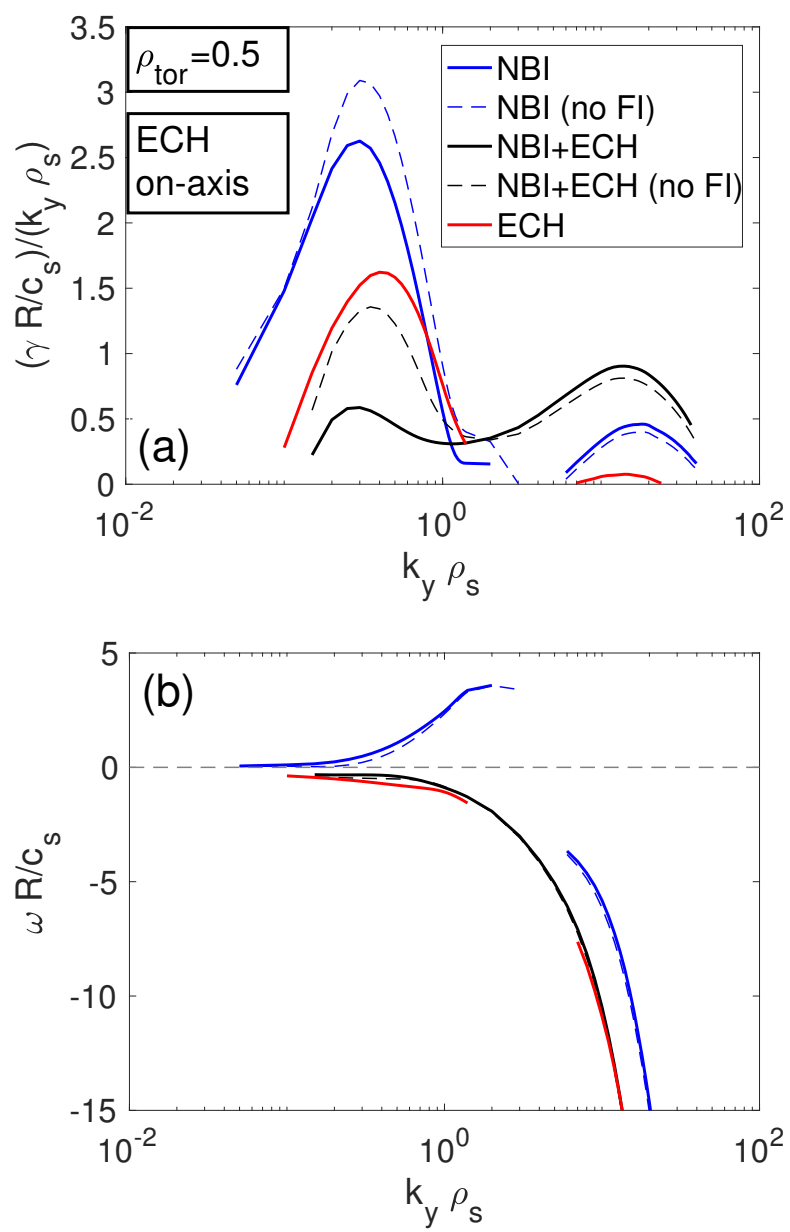

Figure 13. (color online) (a) and (b) $\gamma / k_{y}$ and frequency $\omega$ spectra, respectively. $\gamma$ and $\omega$ are normalised with $c_{s} / R$, while $k_{y}$ is normalised with $1 / \rho_{s}$.

The ETG linear stability thresholds have been estimated by computing growth rates while performing $R / L_{T e}$ scans at fixed $k_{y}$, setting this wave number to the maximum of the electron scale $\gamma$ spectra at mean experimental values of $R / L_{T e}$. The effect of FI has been singled out. The results, compared with the analytic thresholds given by Eq. 1 which have been evaluated setting $Z_{\text {eff }}=2$, are shown in Fig.14. The agreement between the GK and analytic estimates is good, validating the analytic formula in our case. The effect of FI is small. This was expected, since Fig.13 already shows a small effect of FI on electron scale $\gamma$.

\section{B. nonlinear results: comparison of ion-scale GK fluxes with experimental ones}

A set of ion-scale non-linear flux-tube GK simulations have been performed at $\rho_{\text {tor }}=0.5$ to compare with the experimental fluxes (Fig.6 (a) and Fig.9). The results are shown in Fig.15

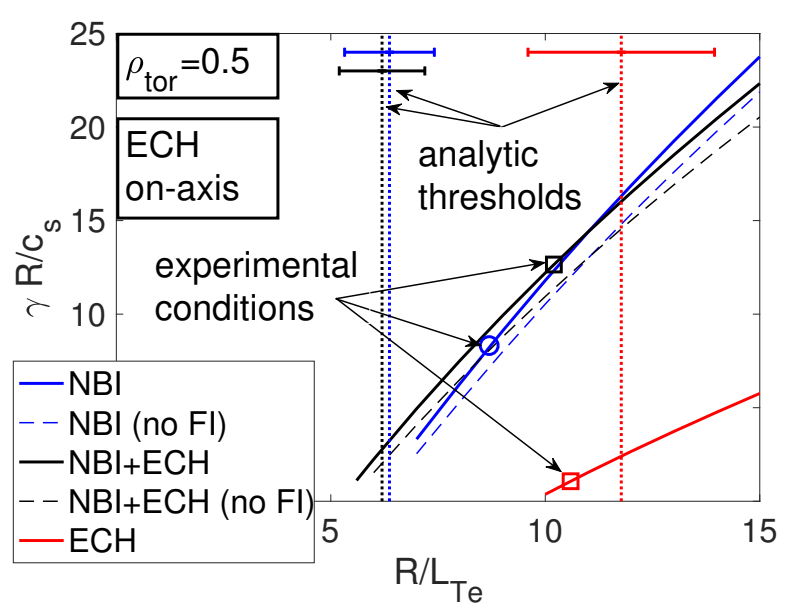

Figure 14. (color online) Growth rate $\gamma_{E T G, \max } R / L_{T e}$ scans. $\gamma_{E T G, \max }$ is the growth rate computed by setting $k_{y}$ equal to the one corresponding to the maximum of the ETG spectrum at mean experimental parameters. The values at experimental $R / L_{T e}$ are indicated by square markers, while the dotted vertical lines represent the analytic ETG thresholds given by Eq.1, obtained setting $Z_{\mathrm{eff}}=2$. Error bars are provided similarly to Fig.6 (a).

(a). For the phases with ECH, only the cases with ECH onaxis have again been considered, while a second simulation has been run for each of them with the lower $R / L_{T e}=7$ value, keeping other parameters constant, in order to give an estimate of the stiffness due to TEM turbulence. One should thus note that the 'low- $R / L_{T e}$ ' GK results should not be directly compared with the experimental data corresponding to $\mathrm{ECH}$ deposition off-axis, where the mean parameters are in general different.

The main result of this section is that the ion-scale GK electron heat flux $q_{e}$ strongly underestimates the experimental value for the mixed NBI/ECH case, implying the need of a contribution from electron scales (ETGs). On the contrary, for the $\mathrm{ECH}$ case the GK $q_{e}$ slightly overestimates the experimental one. For the NBI case, GK $q_{e}$ strongly overestimates the experimental value, probably due to the fact that the ionscale turbulence is dominantly ITG (sensitive to $R / L_{T i}$ ) and this phase shows a large $R / L_{T i}$ value at $\rho_{\text {tor }}=0.5$ (see Table I) with a corresponding large error bar on $T_{i}$ (see Fig.4 (e)). Indeed, $q_{e}$ decreases by $33 \%$ when reducing $R / L_{T i}$ by only $20 \%$, as is shown by the light blue point in Fig. 15 (a). It is worth pointing out that all these flux-tube GK fluxes are 'upper boundaries', since finite Larmor radius effects (also called 'global' effects) could play a significant role in decreasing the fluxes with respect to flux-tube estimates in small-sized machines like TCV [30, 31]. It is thus plausible that global effects could contribute in improving the agreement between GK and experiment for the NBI only case. The analysis of such effects is beyond the scope of this work given the numerical cost of such global simulations. Finally, it shall be noted that this does not invalidate the result that ETGs are needed to explain the experimental electron heat flux for the mixed NBI/ECH case, since the 'upper boundary' $q_{e}$ which has been computed with the flux-tube GK simulation is already strongly underes- 
timating the experimental value.

In order to single out the effects which contribute to stabilising the ion-scale turbulence for the mixed NBI/ECH case, leading to the underestimation of the experimental flux, three additional ion-scale NL GK simulation have been performed, removing $E \times B$ shear, FI and both of them respectively. The results are shown in Fig.15 (b), compared with the experimental value. The results indicate that the FI alone are able to explain the underestimation of $q_{e}$ by GK. Indeed, while removing only the $E \times B$ shearing the electron heat flux still underestimates the experimental one, removing only FI $q_{e}$ is found in agreement with the experiment within error bars. Moreover, the synergic interplay of FI and $E \times B$ which was found in the linear results of section IV A is confirmed by NL ones. The stabilising effect of $E \times B$ shearing is larger with FI ( $\left[q_{e}\right]_{\mathrm{FI}, \mathrm{E} \times \mathrm{B}} /\left[q_{e}\right]_{\mathrm{FI}, \mathrm{NO} \mathrm{E} \times \mathrm{B}}=29 \%$, while $\left.\left[q_{e}\right]_{\mathrm{NO} \text { FI,E } \times \mathrm{B}} /\left[q_{e}\right]_{\mathrm{NO} \text { FI,NO } \mathrm{E} \times \mathrm{B}}=52 \%\right)$, consistently with the larger ratio $\gamma_{E} / \gamma_{\mathrm{i}, \max }$ of the $E \times B$ shearing rate to the maximum linear growth rate at the ion scale which is obtained when FI are taken into account. Finally, it is worth noting that removing both FI and $E \times B$ shear effect, GK overestimates $q_{e}$ [GB]. For completeness, the corresponding NL $k_{y}$ spectra are shown in Fig. 15 (c), indicating that the two stabilising effects are both almost uniform in $k_{y}$.

Coming back to Fig.15 (a), the comparison of the GK TEM stiffness (i.e. the slope of the segment that connects the GK $q_{e}$ [GB] with the one that has been obtained with setting $R / L_{T e}=7$ ) with the experimental one (i.e. the slope of the segment that connects the experimental fluxes with ECH onaxis with the corresponding ones with ECH off-axis) shows a good qualitative agreement for the pure ECH case, in agreement with the picture of section III, while it confirms the negligible role of TEMs for the mixed NBI/ECH case.

Given the lower numerical cost of GK simulations for $\mathrm{ECH}$ only cases (no FI, no $E \times B$ shear) with respect to mixed $\mathrm{NBI} / \mathrm{ECH}$ ones, it was possible to afford two additional NL simulations within our computational resources, corresponding to ECH on-axis cases at the larger radius $\rho_{\text {tor }}=0.7$, in order to verify the presence of ETGs which was predicted in ASTRA fits in Section III. Unexpectedly, the ion-scale NL GK flux does not underestimate the experimental one (see Fig.16 (a)). As a consequence, an alternative picture could be proposed for the ECH only case, where the ETGs are replaced by stiffer TEMs at larger radii. The corresponding $q_{e}$ [GB] vs $R / L_{T e}$ (ASTRA ) is represented in Fig.16 (a) as a solid line, compared with the ETG scenario (dashed). With this new alternative picture, it is still possible to match the experimental phases and amplitudes of the ECH modulation with a good approximation (see Fig.16 (c) and (d)), but it is not possible to recover the experimental flattening of the phases for $\rho_{\text {tor }} \gtrsim 0.5$. The slope of the TEM-driven transport is compared between ASTRA and GK, by running a second GK simulation at $\rho_{\text {tor }}=0.7$ with the lower $R / L_{T e}=7$ value and comparing the ASTRA $q_{e}[\mathrm{~GB}]$ vs $R / L_{T e}$ slope in the 'stiff TEM' picture with the slope of the segment which links the two GK points. The agreement is good. It would be misleading to infer from this that the 'TEM picture' is better than the 'ETG picture'. Given that ion-scale GK simulations are by construction un- able to resolve ETGs and therefore the ETG-driven transport stiffness, this test has been performed to consistently test the ASTRA-GK agreement within the same 'TEM picture'.

Summing up, it is not possible to draw conclusions as to the role of ETG turbulence for the ECH only case with only the available information. In order to test if the GK $q_{e}[\mathrm{~GB}]$ accidentally explains the experimental one at $\rho_{\text {tor }}=0.7$ because we are neglecting other effects such as global ones, one would need to perform very costly NL global simulations, which are out of the scope of this work.
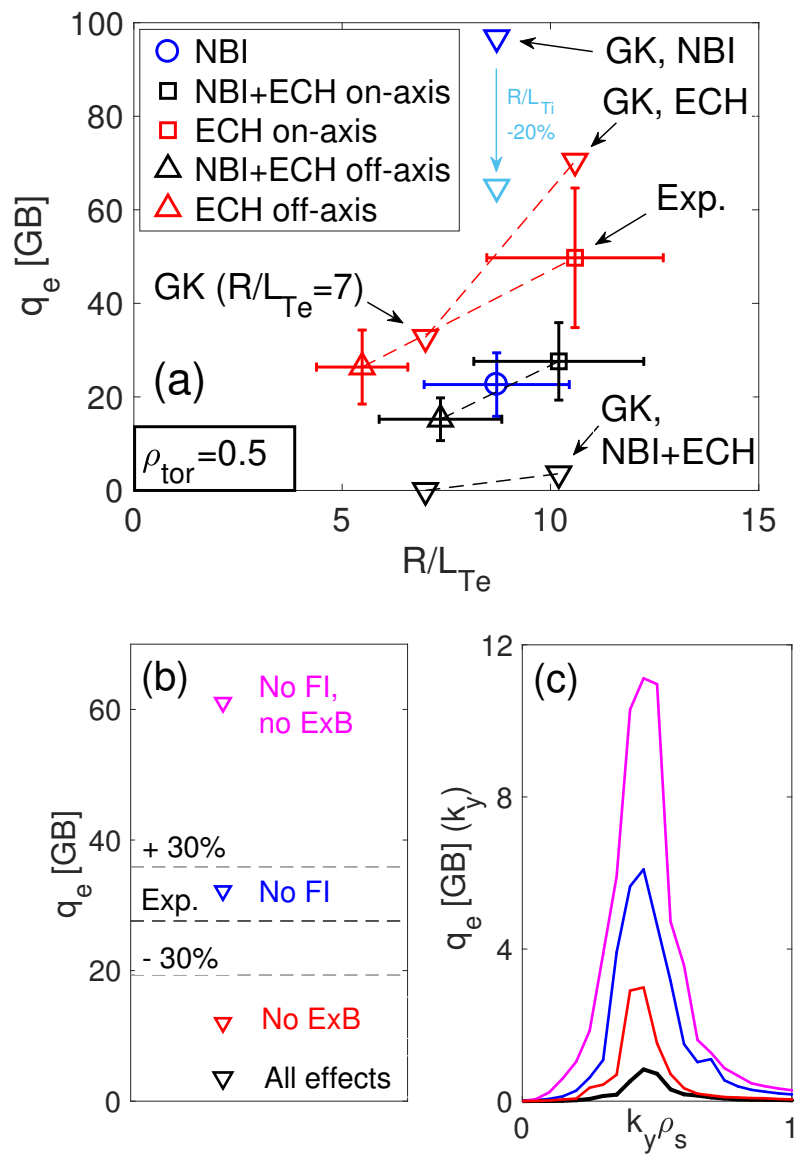

Figure 15. (color online) (a) Ion-scale NL GK heat fluxes in GB units $q_{e}$ [GB] (down-pointing triangles) compared with experimental values (same as in figure (b)). For the ECH and NBI+ECH cases, the $\mathrm{ECH}$ on-axis case parameters have been considered for the GK simulations, while the results at $R / L_{T e}=7$ have been obtained keeping the other parameters constant; (b) Comparison of GK $q_{e}[\mathrm{~GB}]$ with the results obtained removing $E \times B$ shear, FI and both of them respectively; (c) $q_{e}[\mathrm{~GB}]\left(k_{y}\right)$ spectra corresponding to (b) (same color code), defined as $q_{e}=\sum_{k_{y}} q_{e}\left(k_{y}\right)$.

\section{CONCLUSIONS}

A detailed analysis of the electron heat transport has been performed on dedicated TCV discharges, in order to investigate the impact of ETG modes, comparing the experimen- 

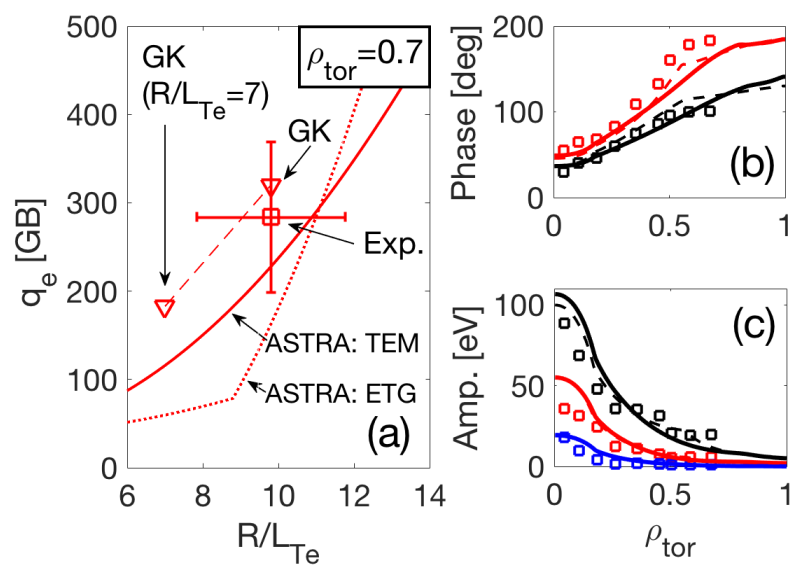

Figure 16. (color online) (a) Comparison of the ASTRA $q_{e}$ [GB] versus $R / L_{T e}$ with the experimental values at $\rho_{\text {tor }}=0.7$, for the ECH on-axis case. Two different ASTRA simulation outputs corresponding to stable ETGs with 'stiff TEMs' (solid) and unstable ETGs (dashed) are compared. The ion-scale GK results are superimposed. (b) and (c) Phase and amplitude of the heat wave (ECH modulation), for the new ASTRA simulation corresponding to stable ETGs with 'stiff TEMs' (solid) and unstable ETGs (dashed). ASTRA results (lines) are compared with experimental data (square markers). Black, red and blue indicate the $1^{\text {st }}, 2^{\text {nd }}$ and $3^{\text {rd }}$ harmonic, respectively.

tal results with GENE gyrokinetic simulations. Cases with different NBI/ECH proportion are compared, also varying the ECH deposition from on-axis to off-axis, in order to explore the transport behaviour with different combinations of $R / L_{T e}$ and $T_{e} / T_{i}$, which are related to ETG stability properties. The threshold and the stiffness of the electron heat flux at mid-radius have been independently estimated by means of a steady state $R / L_{T e}$ scan and a perturbative analysis of the heat wave produced by ECH modulation and ST propagation. While the pure ECH cases show a moderate stiffness, consistent with TEM dominated transport, the mixed NBI/ECH cases show a mixed TEM/ETG transport, which is ETG dominated (moderate/large stiffness) for the case with ECH deposition on-axis, while it is TEM dominated (moderate stiffness) for the case with ECH off-axis. Moreover, at the larger radius $\rho_{\text {tor }}=0.7$ both ECH and mixed NBI/ECH cases are found above ETG threshold, independently of the ECH deposition radius, showing large stiffness.

Linear GK results at mid-radius indicate that at mean experimental parameters ETGs are linearly unstable for all the considered cases (only cases with ECH deposition on-axis have been considered in the GK analysis), but ETGs should significantly contribute to the transport only in the mixed $\mathrm{NBI} / \mathrm{ECH}$ case, consistently with the experimental analysis. At ion scales, TEM are the main modes for the cases with $\mathrm{ECH}$, while ITG are dominant in the NBI only case. Linear results indicate that $E \times B$ shearing is foreseen to have the largest impact on transport for the mixed NBI/ECH case when FI are taken into account. The linear ETG thresholds have been computed by performing $R / L_{T e}$ scans of linear GK simulations, with results which are in good agreement with the analytical estimate [18].
Ion-scale NL simulations show that the experimental $q_{e}$ [GB] is well matched by GK for the ECH only case, while GK strongly underestimates the experimental flux in the mixed $\mathrm{NBI} / \mathrm{ECH}$ case, invoking a possible contribution of ETGs. The main mechanism which is responsible of the GK ion-scale stabilisation is the presence of FI, due to NBI. A larger role of $E \times B$ shearing in heat transport stabilisation is observed when FI are accounted for, consistently with the linear predictions. Finally, the GK flux overpredicts the experimental one for the pure NBI case. The sensitivity of the ITG dominated ion scales to $R / L_{T i}$ within error bars, together with possible global effects, are invoked as possible explanations. An estimate of the $q_{e}$ stiffness due to ion scales only (TEM dominated) has been obtained for the cases with $\mathrm{ECH}$, repeating the GK NL simulations with the reduced $R / L_{T e}=7$ value and looking at the slope of $q_{e}[\mathrm{~GB}] \mathrm{vs} R / T e$. This estimate is in good qualitative agreement, for the pure $\mathrm{ECH}$ case, with the 'TEM stiffness' which can be inferred by comparing results with ECH deposition on- vs off- axis, while it confirms that TEMs almost do not contribute to transport for the NBI+ECH on-axis case.

Due to their reduced numerical cost (no FI, no $E \times B$ ) the NL simulations have been repeated for the ECH only case at the larger radius $\rho_{\text {tor }}=0.7$, where ETGs were expected to play a role according to modulation analysis. However, the ion-scale GK fluxes are sufficient to explain the experimental ones also at this radius. This suggests a possible alternative picture, where ETGs are substituted for the ECH only case by 'stiffer TEM' at larger radii. Nevertheless, it is not possible to discriminate between these two alternative pictures with the available information.

Summing up, the indication of a possible contribution of ETGs to electron heat transport in TCV plasmas comes from both experimental data and GK modelling. The impact of ETGs should be stronger in the mixed NBI/ECH cases due to the high values of $R / L_{T e}$ (due to ECH) which are obtained in conjunction with high values of $T_{i} / T_{e}$ (due to NBI). From the experimental point of view, a valuable addition planned for future experiments will be to use an upgraded Phase Contrast Imaging diagnostic to measure turbulence at the electron scales. On the modelling side, to obtain a confirmation that the missing electron heat flux in the NBI+ECH case is indeed by ETGs, NL multi-scale GK simulations would be needed. An estimate of the global effects will also have to be obtained, to improve the reliability of the comparison of GK with experimental results. Finally, given the large impact of FI on the electron heat transport by stabilisation of the ionscale microturbulence, a study of the effect of assuming the real anisotropic FI velocity distributions instead of the simplified Maxwellian ones, should be pursued. FI should be always modelled taking into account the $E \times B$ shearing, given their interplay. Nevertheless, even if a different FI description could lead to a better agreement of the gyrokinetic flux levels with the experiment, the observed electron stiffness for the mixed NBI/ECH case can not be explained by TEM transport alone, still invoking a possible contribution from the electron scales. 


\section{ACKNOWLEDGEMENTS}

This work has been carried out within the framework of the EUROfusion Consortium and has received funding from the Euratom research and training programme 2014-2018 and 2019-2020 under grant agreement number 633053. The views and opinions expressed herein do not necessarily reflect those of the European Commission. This work was supported in part by the Swiss National Science Foundation. The numerical simulations have been carried out on the MARCONI cluster at CINECA. We thank Antoine Merle (SPC-EPFL, Lausanne) for the help with the TCV magnetic equilibrium reconstruction.

\section{REFERENCES}

[1] W. Horton, Rev. Mod. Phys. 71, 735 (1999).

[2] W. Dorland, F. Jenko, M. Kotschenreuther, and B. N. Rogers Phys. Rev. Lett. 85, 5579 (2000).

[3] F. Jenko and W. Dorland, Phys. Rev. Lett. 89, 225001 (2002).

[4] T. L. Rhodes, W. A. Peebles, M. A. Van Zeeland, J. S. deGrassie, R. V. Bravenec, K. H. Burrell, J. C. DeBoo, J. Lohr, C. C. Petty, X. V. Nguyen et al., Phys. Plasmas 14, 056117 (2007).

[5] A. D. Gurchenko and E. Z. Gusakov, Plasma Phys. Control. Fusion 52, 124035 (2010).

[6] E. Mazzucato, D. R. Smith, R. E. Bell, S. M. Kaye, J. C. Hosea, B. P. LeBlanc, J. R. Wilson, P. M. Ryan, C.W. Domier, N. C. Luhmann et al., Phys. Rev. Lett. 101, 075001 (2008).

[7] D. R. Smith, S. M. Kaye, W. Lee, E. Mazzucato, H. K. Park, R. E. Bell, C.W. Domier, B. P. LeBlanc, F. M. Levinton, N. C. Luhmann et al., Phys. Rev. Lett. 102, 225005 (2009).

[8] G. Wang, W. A. Peebles, T. L. Rhodes, J. C. DeBoo, G. M. Staebler, J. C. Hillesheim, Z. Yan, G. R. McKee, C. C. Petty, W. M. Solomon et al., Phys. Plasmas 18, 082504 (2011).

[9] A. J. Brizard and T. S. Hahm, Rev. Mod. Phys. 79, 421 (2007) .

[10] N. Bonanomi, P. Mantica, G. Szepesi, N. Hawkes, E. Lerche, P. Migliano, A. Peeters, C. Sozzi, M. Tsalas, D. Van Eester, Nucl. Fusion 55, 113016 (2015).

[11] N. T. Howard, C. Holland, A. E. White, M. Greenwald and J. Candy, Phys. Plasmas 21, 112510 (2014).

[12] S.P. Smith, C.C. Petty, A. E. White, C. Holland, R. Bravenec, M. E. Austin, L. Zeng and O. Meneghini, Nucl. Fusion 55, 083011 (2015)

[13] T. Görler and F. Jenko, Phys. Rev. Lett. 100, 185002 (2008).

[14] S. Maeyama and Y. Idomura, Phys. Rev. Lett. 114, 255002 (2015).

[15] N. T. Howard, C. Holland, A. E. White, M. Greenwald and J. Candy, Nucl. Fusion 56,014004 (2016).

[16] N. Bonanomi, P. Mantica, J. Citrin, T. Goerler, B. Teaca and JET Contributors, Nucl. Fusion 58, 124003 (2018).

[17] F. Hofmann, J. B. Lister, M. Anton, S. Barry, R. Behn, S. Bernel, G. Besson, F. Bühlmann, R. Chavan, M. Corboz et al.,
Plasma Phys. Control. Fusion 36, B277 (1994).

[18] F. Jenko, W. Dorland and G. W. Hammett, Phys. Plasmas 8, 4096 (2001).

[19] F. Jenko, W. Dorland, M. Kotschenreuther and B. N. Rogers, Phys. Plasmas 7, 1904 (2000).

[20] T. Görler, X. Lapillonne, S. Brunner, T. Dannert, F. Jenko, F. Merz, D. Told, J. Comput. Phys. 230, 7053 (2011).

[21] G. V. Pereverzev, P. N. Yushmanov, ASTRA - Automated System for TRansport Analysis, Max-Planck-Institut Für Plasmaphysik, IPP-Report, IPP 5/98 (February 2002).

[22] A. H. Kritz, H. Hsuan, R. C. Goldfinger and D. C. Batchelor Conf. Proc., 3rd Int. Symp. on Heating in Toroidal Plasmas ECE, Brussels, Vol. 2, 707 (1982).

[23] O. Sauter, C. Angioni and Y. R. Lin-Liu, Phys. Plasmas 6, 2834 (1999); ibid. 9, 5140 (2002).

[24] Y. Camenen, F. J. Casson, P. Manas, and A. G. Peeters Phys. Plasmas 23, 022507 (2016).

[25] H. Luetjens, A. Bondeson, O. Sauter, Comput. Phys. Commun. 97, 219 (1999).

[26] F. Imbeaux, F. Ryter. and X. Garbet, Plasma Phys. Control. Fusion 43, 1503 (2001).

[27] A. G. Peeters, C. Angioni, M. Apostoliceanu, F. Jenko, F. Ryter, and the ASDEX Upgrade team, Phys. Plasmas 12, 022505 (2005).

[28] A. Jacchia, P. Mantica, F. De Luca, and G. Gorini, Physics of Fluids B: Plasma Physics 3, 3033 (1991).

[29] G. M. Staebler, N. T. Howard, J. Candy and C. Holland Nucl. Fusion 57,066046 (2017).

[30] B. F. McMillan, X. Lapillonne, S. Brunner, L. Villard, S. Jolliet, A. Bottino, T. Görler and F. Jenko, Phys. Rev. Lett. 105, 155001 (2010).

[31] G. Merlo, S. Brunner, O. Sauter, Y. Camenen, T. Görler, F. Jenko, A. Marinoni, D. Told and L. Villard, Plasma Phys. Control. Fusion 57, 054010 (2015). 